\title{
Introducción al análisis y diseño de antenas
}

\author{
Jonnathan López, Ingrid Díaz, Abdiel Sevilla* \\ Universidad Nacional Autónoma de Honduras \\ jonnathan.lopez@gmail.com
}

\begin{abstract}
Resumen
Antennas are structures designed to radiate electromagnetic energy efficiently in a preestablished way by means of design. Without an efficient antenna, energy will be localized and its transmission would not be possible. In this article radiation phenomenon is described, then basic antenna theory is introduced, including characterization of these by a set of parameters. Fasorial notation is introduced conveniently. Moreover, historical context is addressed and the role that these devices have played in the last decades of human history. Lastly, Matlab developed scripts are attached to expose current computational software capacities on antenna analysis and design
\end{abstract}

Keywords: Antennas, radiation, radiation patterns, electromagnetic waves, classical electrodynamics

Las antenas son estructuras diseñadas para radiar energía electromagnética de una manera eficiente y pre-establecida mediante el diseño. Sin una antena eficiente, la energía estaría localizada y la transmisión de energía inalámbrica no sería posible. En este trabajo se describe de forma introductoria el fenómeno de radiación para luego abordar teoría básica de antenas, incluyendo la caracterización de éstas mediante una serie de parámetros. Para ello se introduce la notación fasorial de campos de manera conveniente. Además, se hace referencia al contexto histórico y el papel que han desempeñado estos dispositivos en las últimas décadas. Por último, se incluyen códigos desarrollados en Matlab con el fin de exponer las capacidades de software computacionales actuales para el análisis y diseño de antenas

Palabras clave: Antenas, radiación, patrones de radiación, ondas electromagnéticas, electrodinámica clásica

\section{INTRODUCCIÓN}

工 A definición de antena es variable, por ejemplo, el diccionario de la RAE dicta: "Dispositivo de los aparatos emisores o receptores que, con formas muy diversas, sirve para emitir o recibir ondas electromagnéticas". Algunos autores de teoría electromagnética coinciden en que son estructuras conductoras o dieléctricas mediante las cuales la radiación o emisión de ondas al espacio se cumple eficientemente. Lo cierto es que sin las antenas sería imposible la transmisión de información a través de grandes distancias en la actualidad.
Una antena es una estructura de transición o acoplamiento entre el espacio libre y una línea de transmisión o guía de ondas. El dispositivo guía o línea de transmisión puede tener la forma de cable coaxial o un tubo hueco (guía de ondas), y se utiliza para transportar la energía electromagnética de la fuente a la antena, o desde la antena al receptor. Para el primer caso tendríamos una antena transmisora y para el último una receptora [1]. La figura 1 muestra el esquema general.

El circuito puede ser modelado como lo muestra la figura 2, mediante su equivalente de Thevenin: un generador ideal, una línea de 


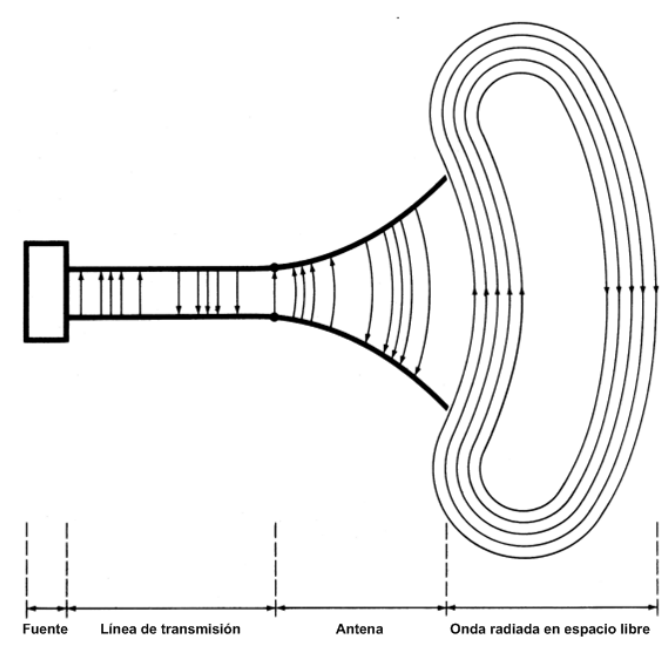

Figura 1: Antena como dispositivo de acoplamiento [1]

transmisión representada por una línea con impedancia $Z_{c}$, y la antena representada por una carga $Z_{A}=\left(R_{L}+R_{r}\right)+j X_{A}$. La resistencia $R_{L}$ representa las pérdidas dieléctricas y de conducción asociadas con la estructura de la antena mientras que $R_{r}$ se refiere a la resistencia de radiación, la cuál representa la radiación de la antena y estudiaremos en la sección VI de este artículo. La reactancia $X_{A}$ se utiliza para representar la parte imaginaria de la impedancia asociada con la radiación de la antena. En condiciones ideales, la energía generada por la fuente debería ser transferida en su totalidad a la resistencia de radiación $R_{r}$ [1], [2].

Las ondas reflejadas de la interfaz crean patrones de interferencia constructiva y destructiva que conocemos como ondas estacionarias dentro la línea de transmisión. Una onda viajera típica se muestra también en la figura 2.

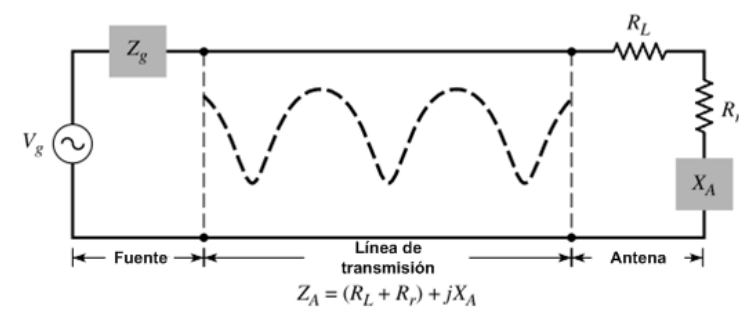

Figura 2: Equivalente de Thevenin, antena emisora [1]
Si las intensidades máximas de campo de la onda viajera son suficientemente grandes, pueden descargas eléctricas dentro de las líneas de transmisión; por ello, el sistema radiante debe ser diseñado apropiadamente.

En sistemas de comunicación inalámbricos avanzados, las antenas también sirven para optimizar o acentuar la energía radiante en algunas direcciones y suprimirlas en otras. Por tanto, una antena también sirve como un dispositivo de direccionamiento. Para sistemas de comunicaciones inalámbricas, la antena es uno de los componentes más críticos. Un buen diseño de la antena puede aminorar los requerimientos del sistema y mejorar el rendimiento general. Un ejemplo típico es un televisor para el cuál la recepción puede ser mejorada mediante antenas de alto rendimiento. Otro caso digno de mencionar es el de las compañias de telefononía móvil en nuestro país que utilizan diferentes tipos de antenas según el ambiente o tipo de cobertura que deseen brindar.

\section{Antecedentes históricos}

La historia de las antenas se remonta a los tiempos de James Clerk Maxwell, con su brillante trabajo de electrodinï¿œmica en 1873. Luego debemos mencionar al profesor Heinrich Hertz quien en 1886 demostró el primer sistema inalámbrico electromagnético aunque únicamente produciendo arcos eléctricos. No fue hasta 1901 cuando Guglielmo Marconi pudo enviar señales sobre largas distancias: una transmisión transatlántica de Inglaterra hasta Canada. Este fue el amanecer de las antenas.

A partir de este notable momento hasta los 1940s, la tecnología de las antenas se basó en elementos radiantes de alambre, que discutiremos en breve, y frecuencias entre $300 \mathrm{MHz}$ a $3 \mathrm{GHz}$ (UHF). La nueva era para las antenas comienza con la segunda guerra mundial cuando surgen nuevos tipos de antenas como las de apertura y reflectoras.

Entre 1960 y el año 2000 se dan grandes avances en la arquitectura y tecnología computacional lo cual tuvo una gran influencia en la ingeniería y diseño de antenas. Se espera un impacto aún mayor en el siglo actual. 


\section{Tipos de antenas}

A continuación presentamos y describimos brevemente los más importantes tipos de antenas.

\section{Antenas de alambre}

Estas antenas son familiares para todos pues las podemos ver en cualquier parte - automóviles, edificios, televisión, aviones, etc. Existen varias formas para éstas antenas tales como alambre recto (dipolo), espira, helicoidal, las cuales se muestran en la figura 3. Las antenas de espira pueden ser de diversas formas. En la sección 4 deducimos las ecuaciones de campo referente para este tipo de antenas.

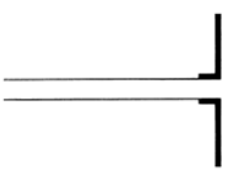

a) Dipolo

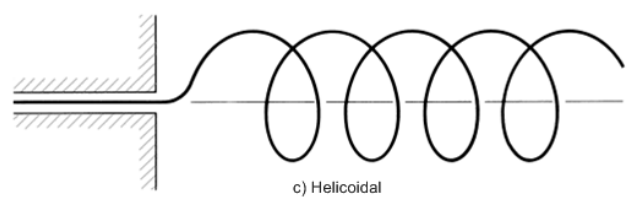

Figura 3: Configuraciones de antena de alambre [1]

\section{Antenas de apertura}

Este tipo de antenas son útiles para altas frecuencias y en aplicaciones tales como aeronaves y naves espaciales pues pueden ser empotradas en las paredes de las aeronaves. Además, se pueden cubrir con materiales dieléctricos para protección de la intemperie. La figura 4 muestra una antena de este tipo.

\section{Antenas reflectoras}

El éxito en la exploración espacial ha dado como resultado grandes avances en teoría de antenas. Dada la necesidad de comunicación a grandes distancias, formas sofisticadas de antenas se deben usar para transmitir y recibir señales que viajan millones de kilómetros. Una forma muy común de este tipo de antena es un reflector parabólico como el mostrado en la figura 4. Se han construido antenas de diámetros de hasta $305 \mathrm{~m}$. Tales dimensiones son necesarias para alcanzar una alta ganancia para recibir y transmitir señales despues de haber recorrido millones de millas [1].
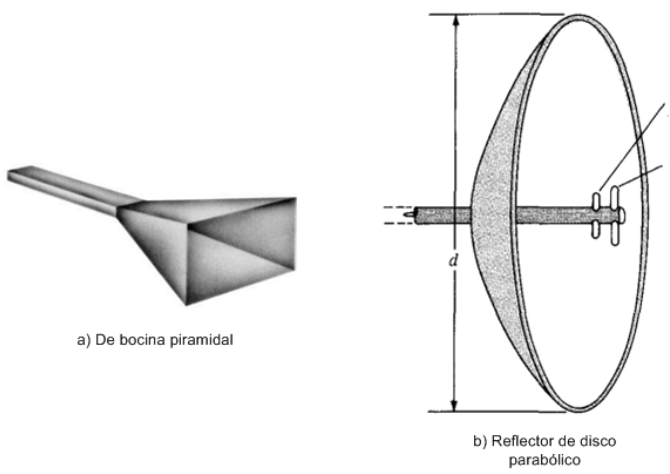

Figura 4: Antenas de apertura y reflectora [1]

\section{Otros tipos de antena}

En la actualidad existen antenas tipo parche o microstrip, las cuales surgieron en los 70s principalmente para aplicaciones espaciales. Estas consisten en un parche metálico sobre un sustrato aterrizado. En la actualidad su uso es tán variado que va desde satélites hasta telófonos móviles.

Otras aplicaciones requieren caracterï¡œsticas de radiación que una sola antena no puede lograr. Es posible realizar una configuración de elementos radiantes o arreglos geométricos y eléctricos que resulte en las caraterísticas de radiación deseadas. Tal es el caso de los arreglos de radiotelecopios como el Very Large Array en Estados Unidos o el Square Kilometre Array en Australia.

\section{EL MECANISMO DE RADIACIÓN}

Una de las primeras preguntas que surge respecto a antenas es: Â¿como se genera la radiación electromagnética? En otras palabras, Â¿como los campos electromagnéticos generados por la fuente, contenidos y guiados por la línea de transmisión y antena, y finalmente "desprendida" de la antena forman una onda en espacio libre? Para responder estas preguntas expondremos de manera cualitativa lo que sucede en una antena de alambre. 
En primer lugar recordemos que para exista radiación requerimos cargas aceleradas o bien corrientes variables en el tiempo. Usualmente nos referimos a corrientes en aplicaciones armónicas en el tiempo mientras que la carga se menciona en transitorios. Consideremos el caso transitorio con un solo alambre. Para crear una aceleración (o desaceleración) el alambre debe ser curvo o discontinuo. Específicamente:

1. Si la carga no se mueve, no se crea corriente y no existe radiación.

2. Si la carga se mueve con rapidez constante:

- No existe radiación si el alambre es recto y finito en extensión.

- Existe radiación si el alambre se curva, dobla o se trunca.

3. Si la carga oscila en el tiempo, radía aún si el alambre es recto.

Un entendimiento cualitativo del fenómeno se obtiene considerando una fuente de pulsos unido a un conductor finito abierto en uno de sus extremos, que puede estar aterrizado mediante una carga discreta. Cuando se energiza inicialmente este alambre, las cargas (los electrones) se ponen en movimiento debido a las líneas de campo eléctrico dentro del conductor. Cuando las cargas se aceleran en el lado de la fuente del alambre y desaleceran durante la reflexión del otro lado, se observa que campos radiantes se producen a cada lado y a lo largo de todo el alambre restante. Radiación más fuerte con un espectro de frecuencia más ancha ocurre si los pulsos son más pequeños o cortos en duraciï̈œn mientras que cargas oscilantes armónicas en el tiempo producen, idealmente, radiación de una solo frecuencia de oscilación. Esto es básicamente el mecanismo responsable de la radiación electromagnética para transitorios (cargas eléctricas como fuente) aunque es posible extenderlo a radiación continua [1].

Hemos hasta ahora descrito el mecanismo de radiación hacia espacio libre. Es preciso, antes de introducir la teoría de campos para antenas, adoptar una notación conveniente para el estudio de estas: la notación fasorial de campos armónicos. Luego entraremos en detalle con antenas elementales y seguidamente se estudian los parámetros básicos de antenas.

\section{FASORES Y CAMPOS ARMÓNICOS}

Antes de iniciar con la descripción de la teoría de antenas, adoptaremos la notación fasorial para campos armónicos en el tiempo, esto es, campos que varían de forma períodica o sinusoidal, ahí su interés práctico. La mayoría de los autores de libros de antenas, por ejemplo [1], 2], [5], utilizan fasores pues simplifica notablemente los cálculos. Además, este análisis se puede extender a la mayoría de formas de onda mediante el análisis de Fourier.

Un fasor $z$ es un número complejo, y como tal, puede expresar en forma rectangular como:

$$
z=x+j y
$$

O bien forma polar:

$$
z=e^{j \phi}=r \not \phi
$$

donde $x$ es la parte real de $z ; y$ es la parte imaginaria de $z ; r$ es la magnitud de $z$, dada por:

$$
r=|z|=\sqrt{x^{2}+y^{2}}
$$

y $\phi$ es la fase dada por:

$$
\phi=\tan ^{-1} \frac{y}{x}
$$

Por otro lado, para convertir la forma polar a rectangular basta aplicar la identidad de Euler:

$$
r e^{j \phi}=r(\cos \theta+j \sin \phi)
$$

Es conveniente efectuar la suma y resta de fasores en forma rectangular, y la multiplicación y división en forma polar. Propiedades básicas:

Suma:

$$
z_{1}+z_{2}=\left(x_{1}+x_{2}\right)+j\left(y_{1}+y_{2}\right)
$$

Resta :

$$
z_{1}-z_{2}=\left(x_{1}-x_{2}\right)+j\left(y_{1}-y_{2}\right)
$$


Multiplicación:

$$
z_{1} z_{2}=r_{1} r_{2} \measuredangle \phi_{1}+\phi_{2}
$$

División:

$$
\frac{z_{1}}{z_{2}}=\frac{r_{1}}{r_{2}} \angle \phi_{1}-\phi_{2}
$$

Raíz cuadrada:

$$
\sqrt{z}=\sqrt{r} \not \phi / 2
$$

Complejo conjugado:

$$
z^{*}=x-j y=r\left\lfloor-\phi=r e^{-j \phi}\right.
$$

Entre otras propiedades importantes. Ahora, introduzcamos el elemento tiempo mediante la expresión:

$$
\phi=\omega t+\theta
$$

donde $\theta$ puede ser una función de coordenadas espaciales o una constante. Siendo así, la parte real e imaginaria de:

$$
r e^{j \theta}=r e^{j} e^{j w t}
$$

están dadas respectivamente por:

$$
\operatorname{Re}\left(r e^{j \phi}\right)=r \cos (\omega t+\phi)
$$

y

$$
\operatorname{Im}\left(r e^{j \phi}\right)=r \sin (\omega t+\phi)
$$

Por ejemplo, consideremos una corriente sinusoidal $I(t)=\cos (\omega t+\phi)$, esta equivale a la parte real de $I_{0} e^{j \theta} e^{j \omega t}$. Del mismo modo una corriente $I^{\prime}(t)=\sin (\omega t+\phi)$ es la parte imaginaria de $I_{0} e^{j \theta} e^{j \omega t}$.

El término complejo $I_{0} e^{j \theta}$, el cual resulta de la eliminación del factor tiempo $e^{j \omega t}$ en $I(t)$ se llama corriente fasorial o corriente de fasor $\mathrm{y}$ lo denotaremos con $I_{s}$ :

$$
I_{s}=I_{0} e^{j \theta}=I_{o} \angle \theta
$$

donde el subíndice $s$ denota que es la forma de fasor de $I(t)$. Así, $I(t)=I_{0} \cos (\omega t+\theta)$, la forma instantánea, puede expresarse como:

$$
I(t)=\operatorname{Re}\left(I_{s} e^{j \omega t}\right)
$$

En general, un fasor puede ser un escalar o un vector. Si un vector $\mathbf{A}(x, y, z, t)$ es un campo armónico en el tiempo, la forma de fasor de $\mathbf{A}$ es $\mathbf{A}_{s}$. La relación entre estas dos cantidades está dada por:

$$
\mathbf{A}=\operatorname{Re}\left(\mathbf{A}_{s} e^{j \omega t}\right)
$$

Por ejemplo, si $\mathbf{A}=A_{0} \cos (\omega t-\beta x) \hat{\mathbf{y}}$, A puede expresarse como:

$$
\mathbf{A}=\operatorname{Re}\left(A_{0} e^{-j \beta x} e^{j \omega t}\right)
$$

Entonces el fasor de $\mathbf{A}, \mathbf{A}_{s}$, es:

$$
\mathbf{A}_{s}=A_{0} e^{-j \beta x} \hat{\mathbf{y}}
$$

Además, notemos que, a partir de la expresión anterior:

$$
\frac{\partial \mathbf{A}}{\partial t}=\frac{\partial}{\partial t} \operatorname{Re}\left(\mathbf{A}_{s} e^{j \omega t}\right)=\operatorname{Re}\left(j \omega \mathbf{A}_{s} e^{j \omega t}\right)
$$

Esto demuestra que tomar la derivada temporal de la cantidad instantánea equivale a multiplicar el fasor por $j \omega$. Esto es:

$$
\frac{\partial \mathbf{A}}{\partial t} \Rightarrow j \omega \mathbf{A}_{s}
$$

De igual modo:

$$
\int \mathbf{A} \partial t \Rightarrow \frac{\mathbf{A}_{s}}{j \omega}
$$

Estamos ya listos para aplicar concepto de fasor a campos electromagnéticos variables en el tiempo. Los campos E, D, H y B, las densidades $\mathbf{J}$ y $\rho_{v}$ y sus derivadas pueden expresarse en forma fasorial. Las ecuaciones de Maxwell en un medio isotrópico, homogóneo y lineal para campos armónicos se resumen en la tabla de la figura 5. Vemos que se ha omitido el factor de tiempo $e^{j \omega t}$, puesto que está asociado con todos los términos y por tanto es redundante, lo que resulta en ecuaciones independientes del tiempo. En esto radica precisamente la justificación del empleo de fasores: el factor de tiempo puede excluirse del análisis de campos armónicos en el tiempo, e incluirse cuando sea necesario [2, [5]. Estamos ya listos para abordar la teoría de campos para las antenas.

\section{Dipolo Hertciano}

En este trabajo centraremos nuestro estudio de campos para antenas de alambre. Comenzaremos con el caso mï œes simple posible e ideal: el dipolo hertciano. Se entiende por dipolo hertciano un elemento de corriente infinitesimal $I$ 


\begin{tabular}{ll}
\hline \multicolumn{1}{c}{ Forma diferencial } & \multicolumn{1}{c}{ Forma integral } \\
\hline$\nabla \cdot \mathbf{D}_{s}=\rho_{v s}$ & $\oint \mathbf{D}_{s} \cdot d \mathbf{S}=\int \rho_{v s} d v$ \\
$\nabla \cdot \mathbf{B}_{s}=0$ & $\oint \mathbf{B}_{s} \cdot d \mathbf{S}=0$ \\
$\nabla \times \mathbf{E}_{s}=-j \omega \mathbf{B}_{s}$ & $\oint \mathbf{E}_{s} \cdot d \mathbf{l}=-j \omega \int \mathbf{B}_{s} \cdot d \mathbf{S}$ \\
$\nabla \times \mathbf{H}_{s}=\mathbf{J}_{s}+j \omega \mathbf{D}_{s}$ & $\oint \mathbf{H}_{s} \cdot d \mathbf{l}=\int\left(\mathbf{J}_{s}+j \omega \mathbf{D}_{s}\right) \cdot d \mathbf{S}$ \\
\hline
\end{tabular}

Figura 5: Forma fasorial de las ecuaciones de Maxwell para campos armónicos [5]

$d l$. Aunque tal elemento de corriente no existe en la realidad, es esencial para calcular por integración el campo de una antena práctica.

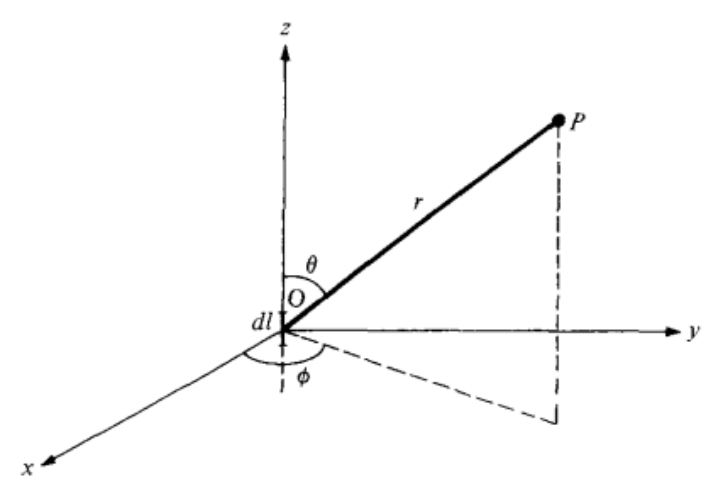

Figura 6: Dipolo hertciano portador de corriente [5]

Considerese el dipolo hertciano que aparece en la figura 6. Supongamos que se ubica en el origen de un sistema de coordenadas y que porta una corriente uniforme (constante a lo largo del dipolo) $I=I_{0} \cos \omega t$. El potencial magnético vectorial retardado debido al dipolo en el punto del campo $P$ está dado por:

$$
A=\frac{\mu[I] d l}{4 \pi r}
$$

donde $[I]$ es la corriente retardada dada por:

$$
\begin{aligned}
{[I] } & =I_{0} \cos \omega(t-r / u)=I_{0} \cos (\omega t-\beta r) \mid \\
& =\operatorname{Re}\left[I_{0} e^{j(\omega t-\beta r)}\right]
\end{aligned}
$$

siendo $\beta=\omega / u=2 \pi / \lambda$ y $u=1 / \sqrt{\mu \varepsilon}$. Se dice que la corriente en el punto $P$ es retardada a causa de un retardo de propagación $r / u$ o retardo de fase $\beta r$ de $O$ a $P$. Al sustituir la segunda ecuación en la primera es posible expresar A en forma de fasor como:

$$
A_{z s}=\frac{\mu I_{0} d l}{4 \pi r} e^{-j \beta r}
$$

La transformación de este vector de coordenadas cartesianas en esfericas produce:

$$
\mathbf{A}_{s}=\left(A_{r s}, A_{\theta s}, A_{\phi s}\right)
$$

donde

$A_{r s}=A_{z s} \cos \theta, A_{\theta s}=-A_{z s} \sin \theta, A_{\phi s}=0$

Además, sabemos que $\mathbf{B}_{s}=\mu \mathbf{H}_{s}=\nabla \times \mathbf{A}_{s}$; así, el campo $H$ se obtiene como:

$$
\begin{aligned}
& H_{\phi s}=\frac{I_{0} d l}{4 \pi} \sin \theta\left[\frac{j \beta}{r}+\frac{1}{r^{2}}\right] e^{-j \beta r} \\
& H_{r s}=0=H_{\theta s}
\end{aligned}
$$

El campo $\mathbf{E}$ se haya mediante $\nabla \times \mathbf{H}=\varepsilon \partial \mathbf{E} / \partial t$ o en notación fasorial $\nabla \times \mathbf{H}_{s}=j \omega \varepsilon \mathbf{E}_{s}$ :

$$
\begin{aligned}
& E_{r s}=\frac{\eta I_{0} d l}{2 \pi} \cos \theta\left[\frac{1}{r^{2}}+\frac{j}{\beta r^{3}}\right] e^{-j \beta r} \\
& E_{\theta s}=\frac{\eta I_{0} d l}{4 \pi} \sin \theta\left[\frac{j \beta}{r}+\frac{1}{r^{2}}-\frac{j}{\beta r^{3}}\right] e^{-j \beta r} \\
& E_{\phi s}=0
\end{aligned}
$$

donde $\eta=\frac{\beta}{\omega e}=\sqrt{\frac{\mu}{\varepsilon}}$.

El detenido examen de las ecuaciones de campo revela la presencia de términos que varían entre $1 / r^{3}, 1 / r^{2}$ y $1 / r$. El término $1 / r^{3}$ es el campo electrostático, ya que corresponde al campo de un dipolo eléctrico. Este término domina a los demás en una region muy cercana al dipolo hertciano. El término $1 / r^{2}$ es el campo inductivo, predecible a través de la ley de Biot-Savart. Este término solo es importante en un campo próximo, a distancias cercanas al elemento de corriente. El término $1 / r$ es el campo lejano o remoto o campo de radiación, puesto que es el unico término que permanece en la zona remota; es decir, en un punto muy lejano del elemento de corriente. Aquí nos ocuparemos primordialmente del campo lejano o zona de radiación $(\beta r \gg 1$ o $2 \pi r \gg \lambda)$, donde los términos en $1 / r^{3}$ y $1 / r^{2}$ pueden ignorarse 
en favor del término $1 / r$. Así, en un campo lejano:

$$
\begin{array}{r}
H_{\phi s}=\frac{j I_{o} \beta d l}{4 \pi r} \sin \theta e^{j \beta r}, E_{\theta s}=\eta H_{\phi s} \\
H_{r s}=H_{\theta s}=E_{r s}=E_{\theta s}=0
\end{array}
$$

Cabe señalar, respecto a la ecuación enmarcada, que los términos de radiación de $H_{\phi s} \mathrm{y}$ $E_{\theta s}$ se hayan en la misma fase temporal y son ortogonales, al igual que los campos de una onda plana uniforme. Asimismo, que los campos de la zona próxima y lejana están condicionados a ser desigualdades $\beta r \ll 1$ y $\beta r \gg 1+$, respectivamente. De manera mas especéfica, la frontera entre las zonas próxima y remota (o lejana) esta definida por el valor de $r$, dado por:

$$
r=\frac{2 d^{2}}{\lambda}
$$

donde $d$ es la mayor dimensiï¡œn de la antena. Con estas cantidades de campo, es posible ahora obtener la potencia promedio temporal mediante el vector de Poynting:

$$
\begin{aligned}
\mathbf{P}_{\text {prom }} & =\frac{1}{2} \operatorname{Re}\left(\mathbf{E}_{s} \times \mathbf{H}_{s}{ }^{*}\right) \\
& =\frac{1}{2} \eta\left|H_{\phi s}\right|^{2} \hat{\mathbf{r}}
\end{aligned}
$$

De aquí, es posible demostrar que la potencia radiada promedio temporal, integrando en un cascarón esférico, es:

$$
\begin{aligned}
P_{\text {rad }} & =\int \mathbf{P}_{\text {prom }} \cdot d \mathbf{S} \\
& =\frac{I_{0}{ }^{2} \pi \eta}{3}\left[\frac{d l}{\lambda}\right]^{2}
\end{aligned}
$$

En el vacío, donde $\eta=120 \pi$ :

$$
P_{\text {rad }}=40 \pi^{2}\left[\frac{d l}{\lambda}\right]^{2} I_{0}^{2}
$$

Es conveniente notar que esta potencia equivale a la potencia disipada por la corriente $I=I_{0} \cos \omega t$ en una resistencia ficticia $R_{r} a d$, misma mencionada en la seccion I de este artículo. Es decir:

$$
\begin{aligned}
P_{r a d} & =I_{r m s}^{2} R_{r a d} \\
& =\frac{1}{2} I_{0}^{2} R_{r a d}
\end{aligned}
$$

donde $I_{r m s}$ es el valor de la raíz media cuadradática de $I$, por sus siglas en inglés [5].

\section{Antena DE DIPOLO DE MEDIA ONDA}

El dipolo de media onda debe su nombre a que su longitud equivale a la mitad de una longitud de onda $(l=\lambda / 2)$. Como se observa en la figura 7 , consta de un hilo delgado alimentado o excitado en su centro por una fuente de voltaje conectada a través de una línea de transmisión (una línea de dos alambres, por ejemplo). El campo debido al dipolo puede obtenerse fácilmente si se considera que consiste en una cadena de dipolos hertcianos. El potencial magnético vectorial en $P$ debido a una longitud diferencial $d l(=d z$ en este caso $)$ del dipolo portador de una corriente fasorial $I_{s}=I_{0} \cos \beta z$ es:

$$
d A_{z s}=\frac{\mu I_{0} \cos \beta z d z}{4 \pi r^{\prime}} e^{-j r^{\prime}}
$$
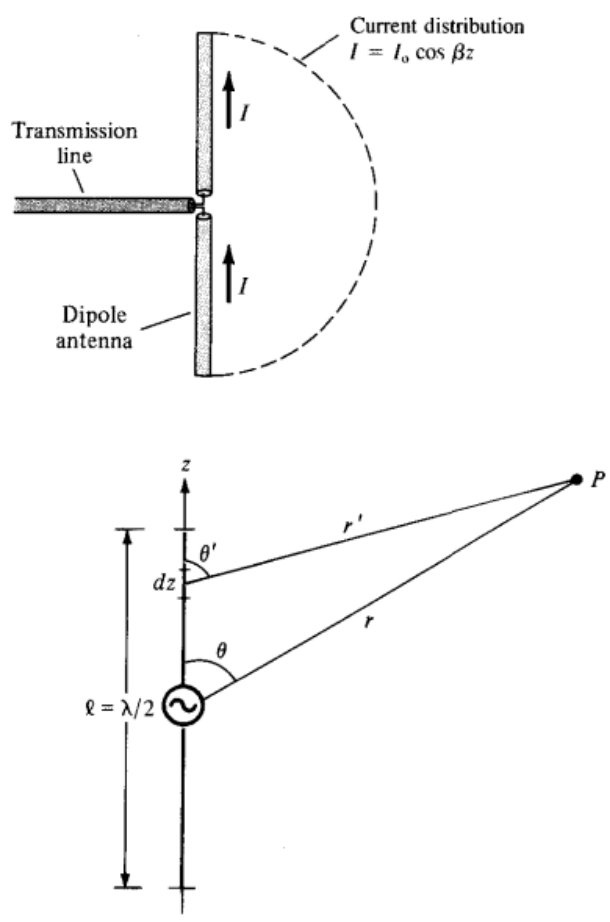

Figura 7: Dipolo de media onda [5]

Notemos que para obtener esta ecuación hemos supuesto una distribución sinusoidal de corriente, puesto que la corriente debe tender a cero en los extremos del dipolo; aunque tambión sería posible una distribución triangular de corriente, los resultados serían menos exactos. La distribución real de corriente de 
la antena no se conoce con precisión; se determina resolviendo las ecuaciones de Maxwell sujetas a las condiciones de frontera en la antena, procedimiento matemático complejo. Sin embargo, el supuesto de la corriente sinusoidal aproxima la distribución obtenido mediante la resolución del problema con valor en la frontera y es de uso frecuente en la teoría de antenas.

Si $r \gg l$ tal como en electrostática:

$$
r-r^{\prime}=z \cos \theta o \quad r^{\prime}=r-z \cos \theta
$$

Así, puede sustituirse $r^{\prime} \approx r$ en el denominador de la ecuación, donde es necesaria la magnitud de la distancia. En cuanto al término de fase en el numerador de la misma ecuación, la diferencia entre $\beta r$ y $\beta r^{\prime}$ es significativa, de manera que $r^{\prime}$ se reemplaza por $r-z \cos \theta$ no por $r$. En otras palabras, el término coseno se mantiene en el exponente y se ignora en el denominador, pues el primero implica la constante de fase y el segundo no. De este modo:

$$
\begin{aligned}
A_{z s} & =\frac{\mu I_{0}}{4 \pi r} \int_{-\lambda / 4}^{\lambda / 4} e^{-j \beta(r-z \cos \theta)} \cos \beta z d z \\
& =\frac{\mu I_{0}}{4 \pi r} e^{0 j \beta r} \int_{-\lambda / 4}^{\lambda / 4} e^{j \beta z \cos \theta} \cos \beta z d z
\end{aligned}
$$

Con base a tablas de integrales:

$$
\int e^{a z} \cos b z d z=\frac{e^{a z}(a \cos b z+b \sin b z}{a^{2}+b^{2}}
$$

Al aplicar esta expresión a la ecuación se obtiene:

$A_{z s}=\frac{\mu I_{0} e^{-j \beta r} e^{j \beta z \cos \theta}}{4 \pi r} \frac{j \beta \cos \theta \cos \beta z+\beta \sin \beta z}{\beta^{2} \cos ^{2} \theta+\beta^{2}}$

Ahora, puesto que $2 \pi / \lambda$ o $\beta \lambda / 4=\pi / 2 \mathrm{y}$ $-\cos ^{2}+1=\sin \theta$, la ecuación se convierte en:

$$
\begin{array}{r}
A_{z s}=\frac{\mu I_{0} e^{-j \beta r}}{4 \pi r \beta^{2} \sin ^{2} \theta}\left[e^{j(\pi / 2) \cos \theta}(0+\beta)-\right. \\
\left.e^{-j(\pi / 2) \cos \theta}(0-\beta)\right]
\end{array}
$$

Del uso de la identidad $e^{j x}+e^{-j x}=2 \cos x$ resulta:

$$
A_{s z}=\frac{\mu I_{0} e^{-j \beta r} \cos \left(\frac{\pi}{2} \cos \theta\right)}{2 \pi r \beta \sin ^{2} \theta}
$$

Del empleo de la primera ecuación junto con el hecho de que $\mathbf{B}_{s}=\mu \mathbf{H}_{s}=\nabla \times \mathbf{A}$ y $\nabla \times \mathbf{H}_{s}=$ $j w \varepsilon \mathbf{E}_{s}$ se obtienen la forma:

$$
H_{\phi s}=\frac{j I_{0} e^{-j \beta r \cos \left(\frac{\pi}{2} \cos \theta\right)}}{2 \pi r \sin \theta}, E_{\theta s}=\eta H_{\phi s}
$$

Observemos de nuevo que los términos de radiación de $H_{\phi s}$ y $E_{\theta s}$ se encuentran en la misma fase temporal y son ortogonales [5].

Por último, tal como procedimos para el dipolo hertciano, podemos calcular la potencia radiada. En este caso resulta una integral complicada, pero de manera aproximada resulta:

$$
\begin{aligned}
P_{\text {rad }} & =\int \mathbf{P}_{\text {prom }} \cdot d \mathbf{S} \\
& \approx 36.56 I_{0}
\end{aligned}
$$

De manera similar podríamos obtener los campo y potencias debido a otras antenas de alambre como ser la antena de un cuarto de onda o bien antenas de cuadro o espira circular; sin embargo, basta con estas dos para los fines de este trabajo. Ver por ejemplo [1] y [5] para el campo debido a otras configuraciones y para otros tipos de antenas.

\section{PARÁmetros de ANTENAS}

En las secciones anteriores se estudiaron los campos debido a antenas en base a las ecuaciones de Maxwell y de manera conveniente en notación fasorial. Presentamos ahora algunas importantes características de una antena como radiador de energía electromagnética.

\section{Patrones de Radiación}

Un patrón de radiación (o patrón de antena) es un diagrama tridimensional de la radiación de la antena de un campo lejano.

El patrón de radiación o patrón de antena está definida como una función matemética o representación gráfica de las propiedades de radiación de una antena como función del espacio de coordenadas. En la mayoría de los casos, el patrón de radiación está determinado en el campo lejano y es representados como una función de la coordenadas directivas. Los patrones de radiación incluyen densidad de flujo de potencia, intensidad de radiación, magnitud del campo, directividad, fase o polarización. 
El diagrama de la amplitud de un componente especificado del campo $E$ es un patrón de campo o patrón de voltaje; el del cuadrado de la amplitud de $E$, un patrón de potencia. Para evitar el trazado del diagrama tridimensional del patrón de antena, se trazan por separado el $\left|E_{s}\right|$ normalizado contra $\theta$ con $\phi$ constante (patrón del plano $E$ o patrón vertical ) y el $\left|E_{s}\right|$ normalizado contra $\phi \operatorname{con} \theta=\pi / 2$ (patrón del plano $H$ o patrón horizontal). La normalización de $\left|E_{s}\right|$ se realiza respecto al valor máximo del $\left|E_{s}\right|$, de modo que el valor máximo del $\left|E_{s}\right|$ normalizado es la unidad.

En el caso del dipolo hertciano, por ejemplo, el $\left|E_{s}\right|$ normalizado se obtiene de la ecuación siguiente:

$$
f(\theta)=|\sin \theta|
$$

Lo cual es independiente de $\phi$. De la ecuación (1) se obtiene el patrón del plano $E$, un diagrama polar de $f(\theta)$ en el que $\theta$ varía de $0^{\circ}$ a $180^{\circ}$, como se muestra en la figura $8 \mathrm{a}$. Nótese que el diagrama es simétrico en torno al eje $z$ $(\theta=0)$. En cuanto al patrón del plano $H$, se fija $\theta=\pi / 2$ para que $f(\theta)=1$, lo cual equivale a un círculo de radio 1 , como se ilustra en la figura $8 b$.
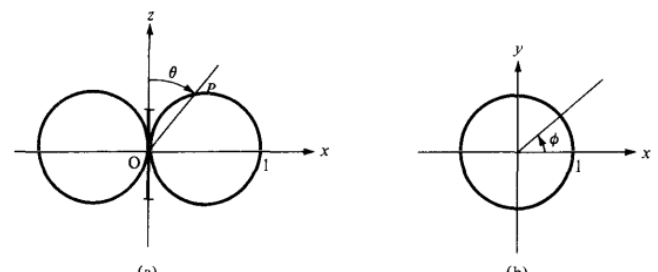

(a)

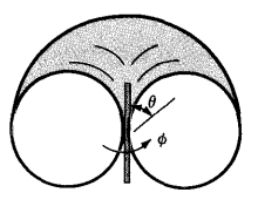

Figura 8: Patrones de radiación dipolo hertciano [1]

El patrón de potencia de la antena es un diagrama de la potencia promedio temporal, $\left|P_{\text {prom }}\right|=$ $P_{\text {prom }}$ con relación a una distancia fija $r$. Esta vez se trazan por separado $P_{\text {prom }}$ contra $\theta$ son $\phi$ constante y $P_{\text {prom }}$ contra $\phi$ con $\theta$ constante.
Con referencia al dipolo hertciano, el patrón de potencia normalizado se obtiene fácilmente de la ecuación (1), en esta forma:

$$
f^{2}(\theta)=\sin ^{2} \theta
$$

lo cual se representa gráficamente en la figura 9. Obsérvese que en las figuras aparecen círculo ya que $f(\theta)$ es independiente de $\phi$, y que el valor de $Q P$ en la figura 9a es la potencia relativa promedio respecto de ese $\theta$ particular. En el punto $Q(\theta=45 \dddot{i} œ)$, así, la potencia promedio equivale a la mitad de la potencia máxima promedio (la cual ocurre en $\theta=\pi / 2$ ).
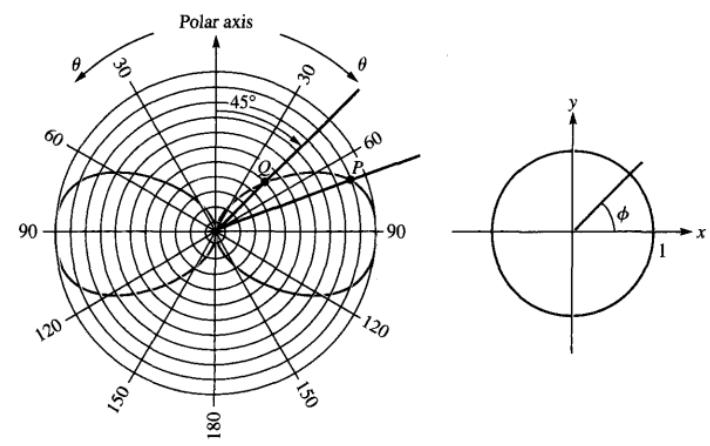

(a)

(b)

Figura 9: Patrones de potencia dipolo hertciano [1]

\section{Intensidad de Radiación}

La intensidad de radiación en una dirección dada está definida como la potencia radiada de una antena por unidad de ángulo sólido. La intensidad de radiación es un parámetro de campo lejano y puede ser obtenido simplemente multiplicando la densidad de radiación por el cuadrado de la distancia.

La intensidad de radiación de una antena se define como:

$$
U(\theta, \phi)=r^{2} W_{r a d}
$$

Donde:

$U(\theta, \phi)=$ Intensidad de radiación ( $\mathrm{W} /$ unidades de ángulo sólido) $W_{\text {rad }}=$ Densidad de radiación $\left(\mathrm{W} / m^{2}\right)$ 
Con base en la ecuación (3), la potencia radiada total promedio puede expresarse como:

$$
\begin{aligned}
P_{\text {rad }}=\oint_{s} W_{\text {rad }} d S & =\oint_{s} W_{r a d} r^{2} \sin \theta d \theta d \phi \\
& =\int_{s} U(\theta, \phi) \sin \theta d \theta d \phi \\
& =\int_{\phi=0}^{2 \pi} \int_{\theta=0}^{\pi} U(\theta, \phi) d \Omega
\end{aligned}
$$

Donde $d \Omega=\sin \theta d \theta d \phi$ es el ángulo sólido $d i$ ferencial, en estereorradianes (sr). De ahí que la intensidad de radiación $U(\theta, \phi)$ se mida en watts por estereorradión (W/sr). El valor promedio de $U(\theta, \phi)$ es la potencia radiada total dividida entre $4 \pi$ sr; es decir:

$$
U_{\text {prom }}=\frac{P_{\text {rad }}}{4 \pi}
$$

\section{Directividad}

Aparte de los patrones de antena anteriormente descritos, a menudo nos interesa cantidades medibles como la ganancia y la directividad para determinar las caracterï¿œsticas de radiaciï;œn de una antena.

La directividad de una antena es definida segï¿œn la IEEE Standard Definitions of Terms for Antennas como "la razón de la intensidad de radiación en una dirección dada de la antena a la intensidad de radiación promediada sobre todas las direcciones". La intensidad de radiación promedio es igual a la potencia radiada total dividida entre $4 \pi$.

La directividad $D(\theta, \phi)$ de una antena es una medida de la concentración de la potencia radiada en una dirección particular $(\theta, \phi)$.

La directividad puede considerarse como la capacidad de una antena para dirigir potencia radiada en una dirección especéfica. Usualmente se le obtiene como la razón de la intensidad de radiación en una dirección dada $(\theta, \phi)$ a la intensidad de radiaciï ¿œn promedio; es decir,

$$
D(\theta, \phi)=\frac{U(\theta, \phi)}{U_{\text {prom }}}=\frac{4 \pi U(\theta, \phi)}{P_{\text {rad }}}
$$

Mediante la sustitución de la ecuación (3) en la ecuación (6) $W_{\text {rad }}$ puede expresarse en términos de ganancia directiva como:

$$
W_{\text {rad }}=\frac{D}{4 \pi r^{2}} P_{r a d}
$$

La directividad $D(\theta, \phi)$ depende del patrón de antena. En el caso del dipolo hetciano (lo mismo que del dipolo $\lambda / 2$ y el monopolo $\lambda / 4)$, se advierte que $W_{\text {rad }}$ es máxima en $\theta=\pi / 2$ y mínima (de cero) en $\theta=0$ o $\pi$. Así, el dipolo hertciano irradia potencia de una dirección transversal a su longitud. Respecto de una antena isotrópica (aquella que irradia por igual en todas direcciones), $D=1$. Sin embargo, esta antena no es real sino ideal.

La directividad máxima $D_{\max }$ de una antena es la razón de la intensidad de radiación máxima a la intensidad de radiaciï ¿œn promedio

$$
D_{\max }=\frac{U_{\max }}{U_{\text {prom }}}=\frac{4 \pi U_{\max }}{P_{\text {rad }}}
$$

Para una antena isotrópica es fácil de ver que $D_{\max }=1$ ya que $U_{\max }$ y $U_{\text {prom }}$ son iguales en magnitud; este es el menor valor que puede adoptar $D_{\max }$.

En cuanto al dipolo hertciano:

$$
D(\theta, \phi)=1.5 \sin ^{2} \theta, D_{\max }=1.5
$$

Para el dipolo de $\lambda / 2$ :

$$
D(\theta, \phi)=\frac{\eta}{\pi R_{\text {rad }}} f^{2}(\theta), D=1.64
$$

Donde $\eta=120 \pi, R_{\text {rad }}=73 \Omega \mathrm{y}$

$$
f(\theta)=\frac{\cos \left(\frac{\pi}{2} \cos \theta\right)}{\sin \theta}
$$

\section{Ganancia de potencia}

Nuestra definición de la directividad no tiene en cuenta la pérdida óhmica de potencia $P_{l}$ de la antena.. $P_{l}$ se debe a que el conductor de que está hecha la antena de de conductividad finita. Como se ilustra en la figura 10 , si $P_{\text {ent }}$ es la potencia de entrada total de la antena:

$$
P_{\text {ent }}=P_{l}+P_{\text {rad }}=\frac{1}{2}\left|I_{\text {ent }}\right|^{2}\left(R_{l}+R_{\text {rad }}\right)
$$

donde $I_{\text {ent }}$ es la corriente en las terminales de entrada, $R_{\text {rad }}$ es la resistencia de radiación y $R_{l}$ la resistencia de pérdida u óhmica de la antena. En otras palabras $P_{\text {ent }}$ es la potencia aceptada por la antena en sus terminales durante el proceso de radiación, y $P_{\text {rad }}$ la potencia radiada por la antena; la diferencia entre ambas es $P_{l}$, la potencia disipada dentro de la antena. 


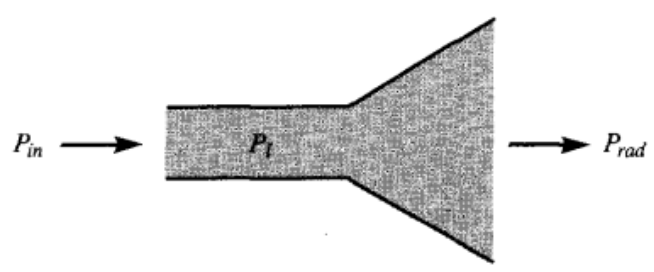

Figura 10: Patrones de potencia dipolo hertciano [1]

La ganancia de potencia $G_{p}(\theta, \phi)$ de la antena se define como:

$$
G_{p}(\theta, \phi)=\frac{4 \pi U(\theta, \phi)}{P_{\text {ent }}}
$$

La razón de la ganancia de potencia en cualquier dirección especificada a la direccionalidad es la eficiencia de radiación $\eta_{r}$ de la antena; esto es:

$$
\eta_{r}=\frac{G_{p}}{D}=\frac{P_{r a d}}{P_{\text {ent }}}
$$

La introducción de la ecuación (12) resulta en:

$$
\eta_{r}=\frac{P_{r a d}}{P_{\text {ent }}}=\frac{P_{\text {rad }}}{R_{\text {rad }}+R_{l}}
$$

En muchas antenas, $\eta_{r}$ se acerca a $100 \%$, de manera que $G_{p} \approx D$.

Directividad y ganancia suelen expresarse en decibelios (dB). Así:

$$
\begin{aligned}
& D(d B)=10 \log _{10} D \\
& G(d B)=10 \log _{10} G
\end{aligned}
$$

Cabe mencionar en este punto que los patrones de radiación de una antena se miden habitualmente en la región del campo lejano, concebida por lo general como existente en una distancia $r \geq r_{\text {min }}$, donde:

$$
r_{\min }=\frac{2 d^{2}}{\lambda}
$$

y $d$ es la mayor dimensión de la antena. Por ejemplo, $d=l$ en la antena de dipolo eléctrico [1], 2].

\section{EJEMPlos - CÁlCUlO DE ANTENAS}

Consideremos ahora un pequeño ejemplo para aplicar las relaciones de campo encontradas en las secciones anteriores. Un punto en $\theta=\mu / 2$ se encuentra a $2 \mathrm{~km}$ de una antena en aire, se precisa de una intensidad de campo magnético de $5 \mu \mathrm{A} / \mathrm{m}$. Sin considerar las pérdidas óhmicas, calculemos la potencia que debe transmitir la antena para los diferentes tipos de antena de alambre que hemos estudiado.

Dipolo hertciano de $\lambda / 25$ de longitud

Para un dipolo Hertciano, vimos que:

$$
\left|H_{\phi s}\right|=\frac{I_{0} \beta d l \sin \theta}{4 \pi r}
$$

donde $d l=\lambda / 25$ o $\beta d l=\frac{2 \pi}{\lambda} \cdot \frac{\lambda}{25}=\frac{2 \pi}{25}$.

Por tanto:

$$
5 \times 10^{-6}=\frac{I_{0} \cdot \frac{2 \pi}{25} \cdot 1}{4 \pi\left(2 \times 10^{-3}\right)}=\frac{I_{0}}{10^{5}}
$$

o bien:

$$
I_{0}=0.5 \mathrm{~A}
$$

Así que:

$$
\begin{aligned}
& P_{\mathrm{rad}}=40 \pi^{2}\left[\frac{d l}{\lambda}\right] I_{0}^{2}=\frac{40 \pi^{2}(0.5)^{2}}{25^{2}} \\
& P_{\mathrm{rad}}=158 \mathrm{~mW}
\end{aligned}
$$

Dipolo de $\lambda / 2$

En este caso, utilizando las relaciones de la sección $\mathrm{V}$ :

$$
\begin{aligned}
\left|H_{\phi s}\right| & =\frac{I_{0} \cos \left(\frac{\pi}{2} \cos \theta\right)}{2 \pi r \sin \theta} \\
5 \times 10^{-6} & =\frac{I_{0} \cdot 1}{2 \pi\left(2 \times 10^{-3}\right) \cdot(1)}
\end{aligned}
$$

O bien:

$$
I_{0}=20 \pi \mathrm{mA}
$$

Por tanto, la potencia radiada requerida es:

$$
\begin{aligned}
P_{\text {rad }} & =\frac{1}{2} I_{0}^{2} R_{\text {rad }}=\frac{1}{2}(20 \pi)^{2} \times 10^{-6}(73) \\
& =144 \mathrm{~mW}
\end{aligned}
$$

Monopolo de $\lambda / 4$ : Aunque no estudiamos esta antena en esta trabajo, es posible obtener de manera similar a las antenas hertcianas y de media onda las cantidades de campo y potencias promedio. Para fines comparativos mostramos los resultados en este caso:

$$
I_{0}=20 \pi \mathrm{mA}
$$


La potencia radiada será:

$$
\begin{aligned}
P_{\text {rad }} & =\frac{1}{2} I_{0}^{2} R_{\text {rad }}=\frac{1}{2}(20 \pi)^{2} \times 10^{-6}(36.56) \\
& =72 \mathrm{~mW}
\end{aligned}
$$

Para una antena de cuadro (ver [5] para las relaciones pertinentes):

$$
\left|H_{\theta s}\right|=\frac{\pi I_{0}}{r} \frac{S}{\lambda^{2}} \sin \theta
$$

En el caso de una vuelta $S=\pi \rho_{0}^{2}$. De modo que para $N$ vueltas $S=N \pi \rho^{2}$. Así:

$$
5 \times 10^{-6}=\frac{\mu I_{0} 10 \pi}{2 \times 10^{3}}\left[\frac{\rho}{\lambda}\right]^{2}
$$

O bien:

$$
\begin{aligned}
I_{0} & =\frac{10}{10 \pi^{2}}\left[\frac{\lambda}{\rho^{2}}\right]^{2} \times 10^{-3} \\
& =40.53 \mathrm{~mA} \\
R_{\text {rad }} & =\frac{320 \pi^{4} S^{2}}{\lambda^{4}}=320 \pi^{6} N^{2}\left[\frac{\rho}{\lambda}\right]^{4} \\
& =320 \pi^{6} \times 100\left[\frac{1}{20}\right]^{4}=192.3 \Omega \\
P_{\text {rad }} & =\frac{1}{2} I_{0}^{2} R_{\text {rad }}=\frac{1}{2}(40.53)^{2} \times 10^{-6}(192.3) \\
& =158 \mathrm{~mW}
\end{aligned}
$$

Examinemos otro ejemplo:

Una antena situada en la superficie de un terreno plano transmite una potencia promedio de $200 \mathrm{~kW}$. Suponiendo que la totalidad de la potencia es emitida de manera uniforme sobre la superficie de un hemisferio con la antena en el centro, calcule el vector de Poynting promedio temporal a $50 \mathrm{~km}$.

La potencia radiada es:

$$
P_{\text {rad }}=\int \mathbf{P}_{\text {prom }} \cdot d \mathbf{S}=P_{\text {prom }} 2 \pi r
$$

Por tanto:

$$
\begin{aligned}
P_{\text {prom }} & =\frac{P_{r a d}}{s \pi r^{2}}=\frac{200 \times 10^{3}}{2 \pi\left(50 \times 10^{6}\right)} \\
& =12.73 \mu \mathrm{W} / \mathrm{m}^{2}
\end{aligned}
$$




\section{Programas en Matlab}

Se presentan a continuación dos códigos desarrollados en Matlab para el cálculo y apreciación de los diferentes parámetros de antenas expuestos en este trabajo. La figura 11 muestra algunos gráficos generados con estos programas. Para el diseño de antenas en la actualidad y producto de los grandes avances computacionales, existen muchos programas especializados. Digno de mención es el software comercial Antenna Magus Antenna Magus o bien el proyecto abierto FDTD Antenna Simulator.

Programa 1: Directividad, resistencia de radiación y potencia radiada

function Dipolo

$\%$ Dipolo

\% Calculo de la Directividad (DO), Resistencia de Radiaciï¿œn (Rrad),

$\%$ y Potencia Radiada (Prad)

$\%$ para una longitud de onda dada (lamda), longitud de dipolo y corriente mï¿œima. $\%$

$\%$ Estos calculos son para una antena de dipolo de cualquier longitud,

$\%$ Centrada en el origen colineal con el eje $z$.

$\%$

lamda $=\operatorname{input}($ 'longitud de onda $($ default=500): ') ;

if isempty (lamda)

lamda $=500 ; \% \% \% \% \% \% \% \% \%$ Valor por defecto de lambda $=500$

end

multiplier = input (' $\backslash \mathrm{n}$ Longitud de la antena como fracci $\backslash\{0\} \mathrm{n}$ de $l a$

longitud de onda \n (.5 para $1 / 2$ onda) \n (.005 para dipolo infinitecimal) $\backslash \mathrm{n}$

(1.25 para ver algunos lobulos laterales) \n Ingrese fracci $\backslash\{0\} n$ (default=.5): ');

if isempty(multiplier)

multiplier $=.5 ; \% \% \% \% \% \% \% \% \%$ valor por defecto del factor de multiplicaci $\backslash\{0\} \mathrm{n}=0.5$

end

ant_length $=$ multiplier*lamda; $\%$ Calculo de la longitud de la antena

current = input (' $\backslash n$ Corriente $m \backslash$ ' $\{$ a\}xima en Amperios (default=1): ');

if isempty (current)

current=1; $\% \% \% \% \% \% \% \% \% \% \% \%$ valor por defecto de la corriente $=1$

end

Prad $=0$;

$\mathrm{T}=[0: 2 * \mathrm{pi} / 360: 2 * \mathrm{pi}]$;

$\mathrm{P}=[0: 2 * \mathrm{pi} / 360: 2 * \mathrm{pi}]$;

$\mathrm{U}=\operatorname{dipoleint} 1(\mathrm{~T}, \mathrm{P}, \mathrm{lamda}$, ant_length, current)

$\%$ Calculo de la potencia radiada total "integrando" todos los valores de

$\%$ intensidad de radiaci $\backslash\{0\}$ m multiplicado por sin(theta)*dTheta*dphi

$\%$ desde theta $=0$ a theta $=$ pi y de phi=0 a $2 *$ pi;

$\%$ las siguientes 4 lineas implementan las eq. 2-13 del texto de Blanis semicircle1= $\sin (\mathrm{T}(1: 181)) ; \%$ enfila $\sin ($ theta)

prad1 $=U(181,1: 181) ; \%$ saca una secciï¿œn plana

prad $1=\operatorname{prad} 1 . *(\mathrm{pi} / 180) . *$ semicircle $1 ; \%$ multiplica por $\sin ($ theta $) *$ dTheta 
Prad $=\operatorname{sum}($ prad 1$) * 2 *$ pi $; \%$ integra sumando luego multiplicando

$\%$ por dPhi desde 0 a $2 *$ pi. Se asume que es un patr $\backslash$ ' $\{0\}$ n omnidirecional.

\%Al igual Prad se calcula usando "loops" para la doble integral.

$\%$ Prad $=0$;

$\%$ for $(i=1: 361)$

$\%$ for $(j=1: 181)$

$\% \operatorname{Prad}=\operatorname{Prad}+U(i, j) * \sin (T(j)) *(p i / 180)^{\wedge} 2$

$\%$ end

$\%$ end

MD=4*pi*U./Prad; \% MD campo de Magnitudes de Directividad normalizadas de acuerdo con $\%$ la eq. 2-16 del texto de Balanis.

D0 $=\max (\max (M D)) ; \%$ La directividad es el valor $m \backslash$ ' $\{$ a $\}$ ximo en este campo.

$\%$ Elevaci $\backslash\{0\}$ n vs. Directividad

M1=MD $(91,:) ; \%$ grafica con theta constante y phi=90

figure; polar(T,M1); title('Plano de Elevaci\'\{o\}n');

$\%$ Azimut vs. Directividad

M2=MD $(:, 91) ; \%$ grafica con phi constante y theta=90

figure; polar(P,M2');title('Plano Azimutal');

$\%$ Plot the whole Directivity Magnitude field.

\%Grafica el campo de magnitud de directividad

figure; mesh(MD);

xlabel ('Elevaci \'\{o\}n');

ylabel ('Azimut');

zlabel ('Intencidad (U)');

title (ant_length/lamda);

$\operatorname{Rrad}=2 * \operatorname{Prad} /$ current $^{\wedge} 2$;

n_tehta $=181 ; \%$ Elevaci $\backslash\{$ o $\} \mathrm{n}$

n_phi $=181 ; \%$ Azimut

[tehta,phi] =meshgrid (0:pi./(n_tehta-1):pi,0:2*pi./(n_phi-1):2*pi) ;

Bas = multiplier;

Num $=\cos ($ pi $*$ Bas $* \cos ($ tehta $))-\cos ($ pi $*$ Bas $) ;$

Den $=\sin ($ tehta $)$;

radio $=$ Num. $/$ Den;

$\%$ radio $=\mathrm{MD}$;

$\%$ Transformando a XYZ

$\mathrm{X}=$ radio $. * \sin ($ tehta $) . * \cos (\mathrm{phi})$;

$\mathrm{Y}=$ radio. $*$ sin (tehta).*sin (phi);

$\mathrm{Z}=\operatorname{radi} 0 . * \cos ($ tehta $)$;

$\%$ Patr $\backslash\{0\} n$ en 3D

figure; $\operatorname{mesh}(\mathrm{X}, \mathrm{Y}, \mathrm{Z})$;

xlabel ('X');

ylabel ('Y');

zlabel (' $\mathrm{Z}$ ') ;

title('patr】' $\{0\} n$ en 3d');

$\%$ axis image;

$\% \% \% \% \% \% \% \% \%$ radiador isotr $\backslash$ ' \{o\}pico 


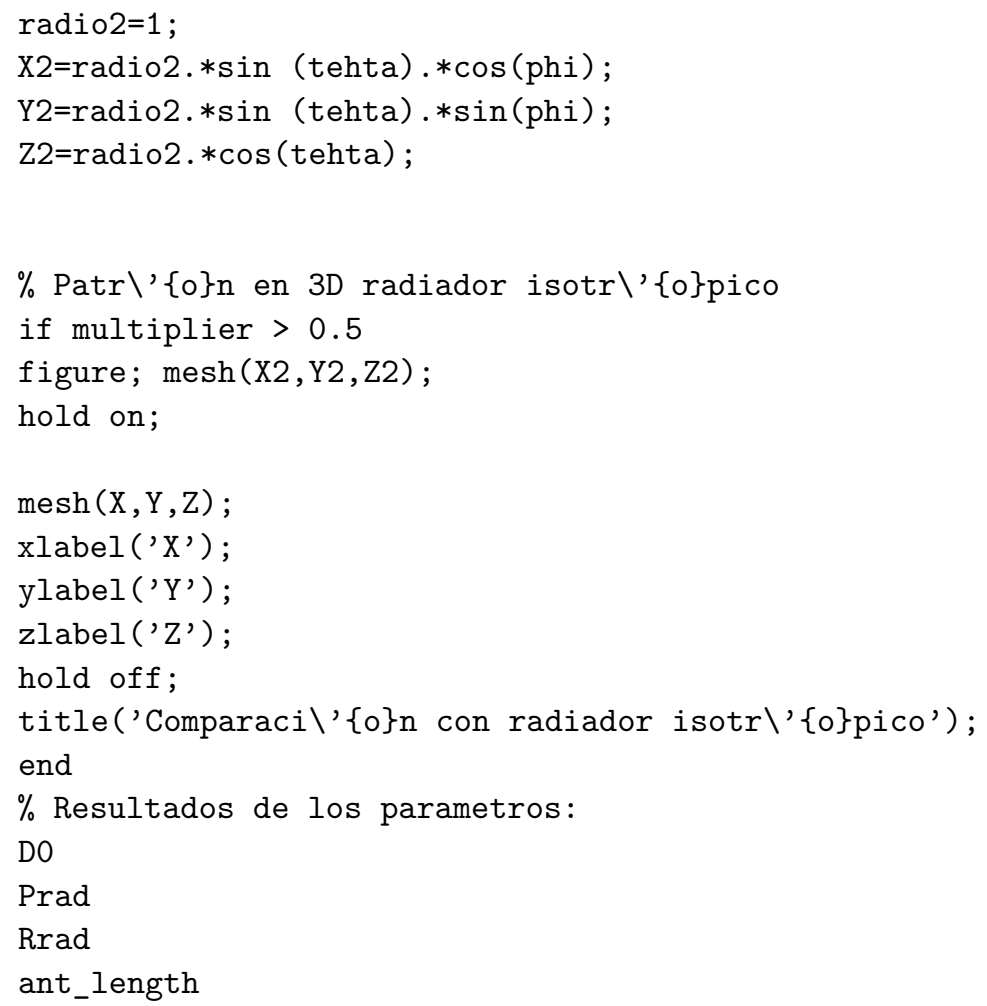

Programa 2: Intensidad de radiación

function $[F]=$ dipoleint (theta, phi, lamda, length1, current)

$\% \mathrm{~F}=$ dipolant (theta, phi, lamda, length1, current)

$\%$ calcula la intensidad de radiaci \'\{o\}n de una antena de dipolo usando las eq.

$\%$ 4-62 en la p\'\{a\}gina 153 y 2-12a en la pag. 38 del texto de Balanis.

$\%$ theta $=\backslash$ ' $\{$ a ngulo de elevaci $\backslash\{$ o $\} n$

$\%$ phi $=\backslash$ ' $\{$ a $\}$ ngulo azimutal

$\%$ lambda = longitud de onda (en metros)

$\%$ length 1 = longitud de la antena (en metros).

$\%$ current $=$ corriente $\mathrm{m} \backslash$ ' $\{$ a $\}$ xima (en amps).

mu0 $=4 *$ pi $* 10^{-}-7 ; \%$ permeabilidad (en newtons/amp`2)

epsilon0 $=8.854187817 * 10^{-}-12 ; \%$ permitividad (in Farads/meter )

eta $=\operatorname{sqrt}(\mathrm{mu} 0 /$ epsilon0); $\%$ impedancia intrinseca (in ohms)

beta $=(2 * \mathrm{pi}) /$ lamda $; \% \mathrm{n} \backslash$ ' $\{\mathrm{u}\}$ mero de onda (in radians $/$ meter)

P1 = ones (size (phi));

[MT MP] = meshgrid(theta, P1);

$\mathrm{n} 1=\cos (($ beta $*$ length $1 / 2) . * \cos (\mathrm{MT}))-\cos ($ beta $*$ length $1 / 2)$;

denom $=(2 *$ pi.$* \sin (\mathrm{MT}+.0000000001))$;

$\% .0000000001$; \% evita el dividir entre 0 asegurandoce que $\sin ()<0$

eTheta $=j *$ eta $*$ current. $*$ n1./denom;

$\%$ ePhi = eTheta $; \%$ ePhi = eta $*$ hPhi and hPhi = eTheta/eta ePhi=(eta/eta)*eTheta

$\%$ ePhi $=0$ para una antena de dipolo.

$\mathrm{ePhi}=0$;

$F=\left(\right.$ abs (eTheta) $\cdot{ }^{\wedge} 2+\operatorname{abs}\left(\right.$ ePhi) $\left.\cdot{ }^{\wedge} 2\right) /(2 * e t a) ;$ 


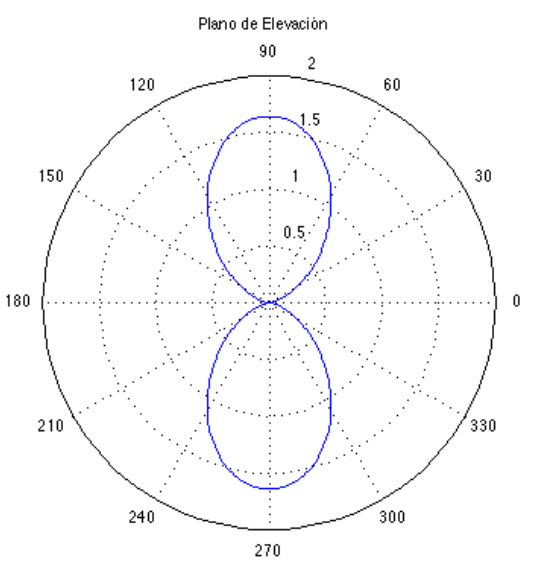

Antena de dipolo de media onda

Plano de elevación

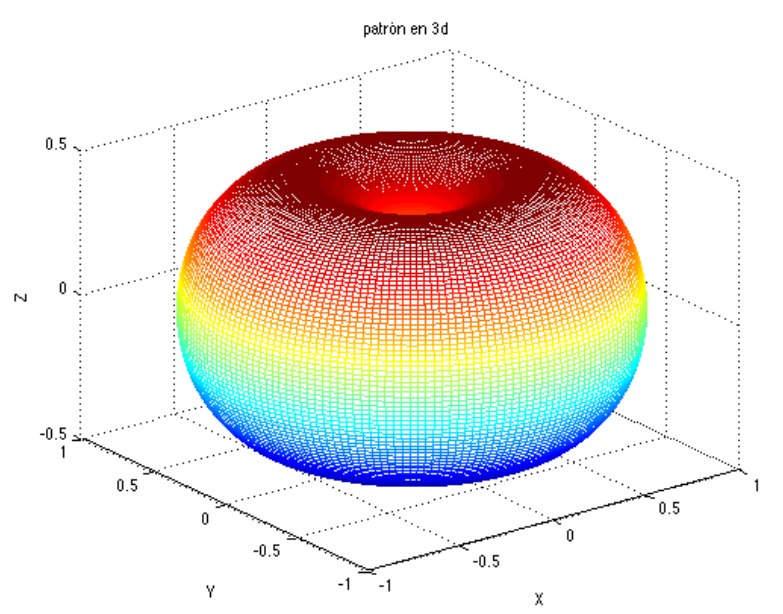

Antena de dipolo de media onda

Patrón 3D, Radiador Isotrópico

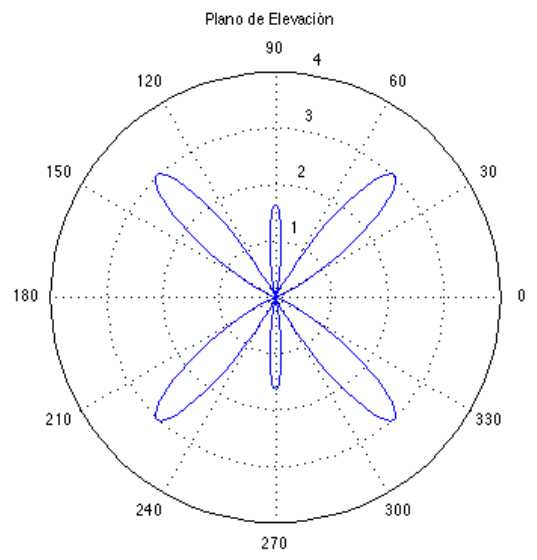

Antena de dipolo de 3 longitudes de onda Plano de elevación

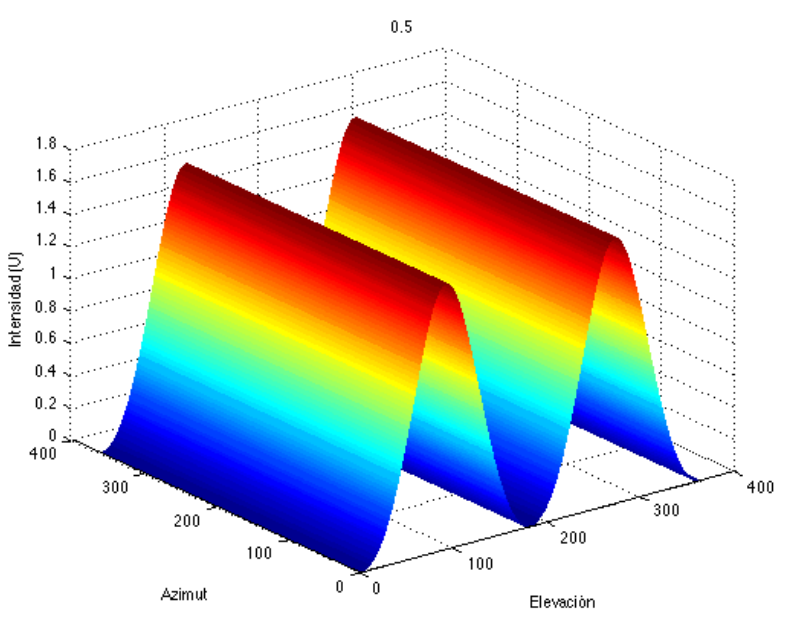

Antena de dipolo de media onda Campo de magnitud de directividad

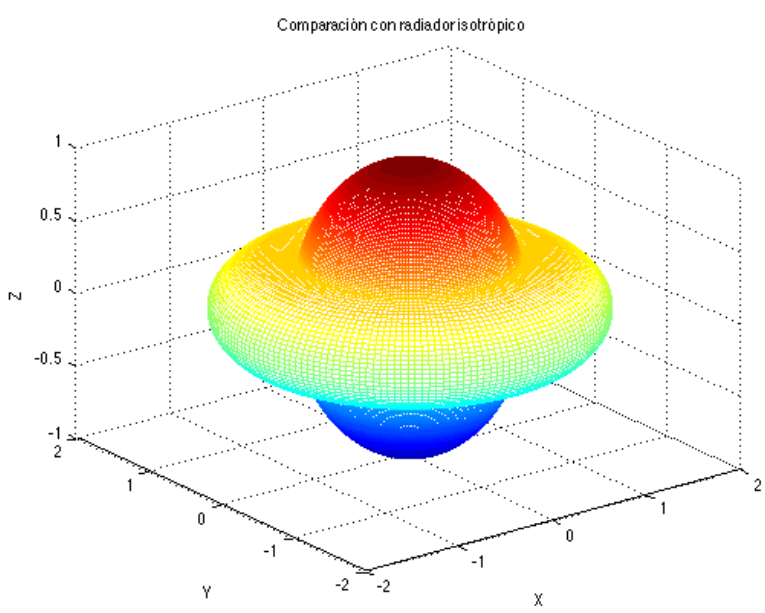

Antena de dipolo de 5/4 de onda Comparación con Radiador Isotrópico

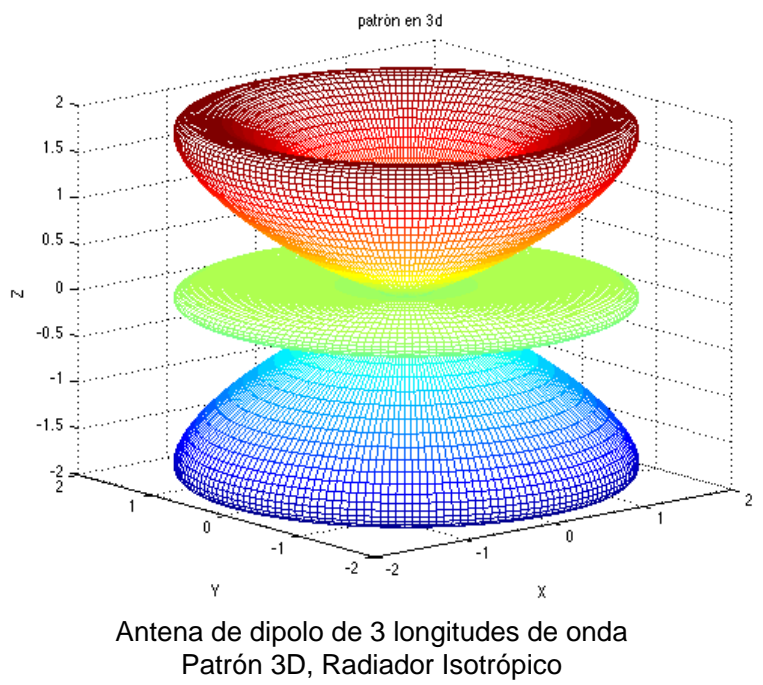




\section{Conclusiones}

- Al proceso de producción de ondas electromagnéticas se le conoce como radiación electromagnética. A las estructuras diseñadas para radiar ondas de manera eficiente se les conoce como antenas emisoras. éstas no radían de la misma manera en todas direcciones, esto es, tienen ciertas propiedades de radiación. Las mismas estructuras se utilizan para extraer energía de una onda electromagnética, en tal caso reciben el nombre de antenas receptoras. Las antenas, emisoras o receptoras, son las cuerdas vocales y oídos de los sistemas de radio comunicación.

- En este trabajo se realizó una breve introducción a la teoría de antenas, comenzando con el papel de las antenas en el contexto histórico, describiendo cualitativamente el proceso de radiación, definiendo el formalismo conveniente para lidear con radiación y antenas - los fasorespara luego encontrar los campos electromagnéticos de algunas antenas sencillas pero de importancia teórica e historica - las antenas de alambre.

- A partir de la teoría clásica de electrodinámica, se introdujeron los parámetros que caracterizan las antenas: directividad, potencia radiada, intensidad de radiación, entre otros, los cuales son elementos fundamentales para el cálculo y diseño de antenas. En ese sentido se presentaron dos diferentes programas en Matlab para tal fin, enfatizando en sus capacidades gráficas; además, se mencionaron softwares computacionales para el diseño de antenas en la actualidad.

\section{REFERENCIAS}

[1] Constantine A. Balanis. Antenna Theory. Wiley Interscience, 2005.

[2] David K. Cheng. Field and Waves Electromagnetics. Addison-Wesley, 1989.

[3] David J. Griffiths. Introduction to Electrodynamics. Prentice Hall, 1999.

[4] John D. Jackson. Classical electrodynamics. John Wiley and Sons, 1962.

[5] Matthew N. O. Sadiku. Elements of Electromagnetics. Oxford Press, 2003.

[6] Warren L. Stutzman and Gary A. Thiele. Antenna Theory and Design. Wiley, 1997.

[7] Roald K. Wangsness. Electromagnetic Fields. Wiley, 1986. 